\title{
NOUVELLE
}

\section{La kinase LKB 1, gardienne de la néoglucogenèse dépendante des acides aminés}

Pierre-Alexandre Just ${ }^{1,2}$, Christine Perret ${ }^{1}$

\author{
${ }^{1}$ Université de Paris, Institut Cochin, Inserm, CNRS, \\ 24 rue du Faubourg Saint-Jacques, 75014 Paris, France. \\ ${ }^{2} \mathrm{AP}-\mathrm{HP}$, Anatomie et cytologie pathologique, \\ Université de Paris, Hôpital Cochin, \\ 27 rue du Faubourg Saint-Jacques, 75014 Paris, France. \\ christine.perret@inserm.fr
}

> L'hyperglycémie chronique est à l'origine des nombreuses complications du diabète de type 2. Une meilleure connaissance des mécanismes de régulation de la glycémie est donc indispensable pour tenter d'établir de nouvelles stratégies thérapeutiques afin de limiter ces complications chez les patients diabétiques réfractaires aux traitements traditionnels. La dérégulation de la néoglucogenèse hépatique, fréquente dans le diabète de type 2 , est un des mécanismes contribuant au développement de I’hyperglycémie [1, 2]. La protéine LKBI (Liver Kinase B1, ou sérine thréonine kinase 11) est un puissant inhibiteur de la néoglucogenèse. Cette kinase contrôle le métabolisme et la croissance cellulaire via l'activation de l'AMPK (AMP-activated kinase) et de 12 autres protéines apparentées, dont les protéines SIK (saltinducible kinases) [3]. L'activation de la voie AMPK favorise les voies métaboliques productrices d'énergie (glycolyse, oxydation des acides gras) aux dépens des voies consommatrices d'énergie (néoglucogenèse et biosynthèse des acides gras). Différentes études ont montré une diminution de cette voie de signalisation dans le foie de souris diabétiques [4]. Bien que l'AMPK ait d'abord été décrite comme étant la principale kinase en aval de LKBI permettant l'inhibition de la néoglucogenèse hépatique, des études plus récentes ont montré que c'était en fait les kinases SIK qui constituaient le principal relai de $L K B I$ dans ce processus $[5,6]$. Ces études ont montré que LKBl contrôlait le programme transcriptionnel néoglucogénique lors des périodes de jeûne, de concert avec le glucagon [6]. Nous avons confirmé le rôle de LKBI dans le contrôle de la néoglucogenèse hépatique à l'aide d'un modèle murin permettant une inactivation ciblée et inductible par le tamoxifène du gène $L K B I$ dans les hépatocytes. Cependant, nos résultats ont montré que la protéine LKBI hépatique, non seulement contrôlait la production de glucose dans le foie, mais était également impliquée dans l'homéostasie énergétique de l'organisme entier [7]. Nous avons en effet observé que ces animaux mutants développaient à plus long terme une sarcopénie ${ }^{l}$ et une cachexie, responsable de leur mort prématurée. L'analyse longitudinale de la composition corporelle de ces animaux par imagerie par résonance magnétique a montré une perte progressive de la masse maigre, qui précédait le développement du phénotype cachectique. De plus, ces souris présentaient une diminution importante de la concentration de nombreux acides aminés dans le sérum, ainsi que dans le foie et le muscle. Comme l'ensemble de ces observations suggérait un rôle plus complexe de LKBI hépatique, non restreint au seul contrôle de l'expression des gènes de la néoglucogenèse, nous avons réalisé une analyse protéomique quantitative «label-free » afin d'identifier des voies métaboliques connexes expliquant le phénotype observé à l'échelle de l'orga-

1 Diminution de la masse musculaire, accompagnée ou non d'une diminution de la force musculaire. nisme entier. Cette analyse a été réalisée sur le foie des souris mutantes et sur celui de souris témoins, en situation de jeûne ou après réalimentation. Elle nous a permis d'identifier le catabolisme des acides aminés comme la voie métabolique majeure (avec celle de la néoglucogenèse) contrôlée par LKBl, suggérant que cette kinase est capable de contrôler l'utilisation des acides aminés comme substrats glucoformateurs, à la fois en situation de jeûne, mais aussi au cours de la période post-prandiale. Par différentes approches complémentaires (tests de tolérance à l'alanine, capacité des hépatocytes primaires à synthétiser du glucose à partir des acides aminés, analyse de flux métabolique à l'aide de $\left[{ }^{14} \mathrm{C}\right]$ alanine sur des explants de foie), nous avons confirmé que les hépatocytes rendus déficients en LKBI avaient effectivement une capacité plus importante à synthétiser de novo du glucose à partir des acides aminés et à le stocker sous forme de glycogène. Ces résultats indiquaient que nous avions identifié un nouveau mécanisme par lequel LKBI exerce sa fonction de suppresseur de la néoglucogenèse hépatique: l'inhibition de l'utilisation des acides aminés provenant du foie et du muscle comme substrat glucoformateur [7].

L'analyse protéomique nous a permis d'identifier un grand nombre d'enzymes du catabolisme des acides aminés, notamment aminotransférases et transporteurs des acides aminés, dont l'expression protéique était contrôlée négativement par LKBI (Figure I). Ce résultat, 


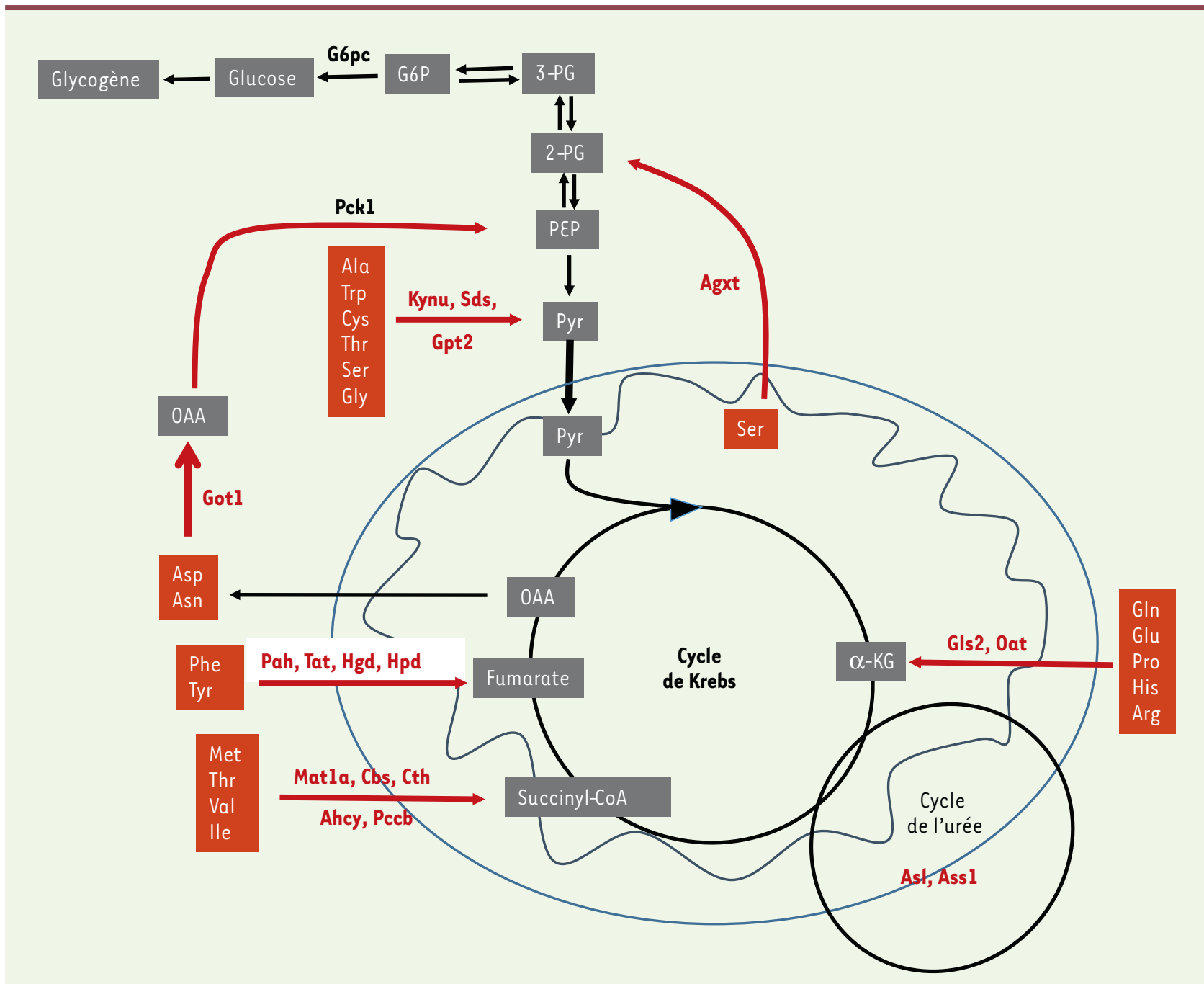

Figure 1. Schéma du catabolisme des acides aminés utilisés comme précurseurs de la synthèse de glucose et impliqués dans le cycle de l'urée. Les acides aminés sont notés dans un cadre rouge. La voie de la glycolyse, le cycle de Krebs (cycle tricarboxylique), ainsi que le cycle de l'urée, sont schématisés. Deux enzymes clés de la néoglucogenèse, Pckl (phosphoénolpyruvate carboxykinase) et G6pc (sous-unité catalytique de la glucose 6-phosphatase), sont également indiquées. Toutes les enzymes notées en rouge sont enrichies dans le protéome des souris mutantes déficientes en LKBI dans le foie, et sont impliquées dans le catabolisme des acides aminés. Gotl : aspartate aminotransférase ; kynu : kynuréninase ; Sds : sérine déshydratase; Gpt2 : alanine aminotransférase 2 ; Agxt : sérine-pyruvate aminotransférase; Gls2 : glutaminase ; 0at : ornithine aminotransférase; Asl : argininosuccinate lyase; Ass l : argininosuccinate synthase ; Matla : adénosylméthionine synthase ; Cbs : cystathionine synthase; Cth : cystathionine gamma lyase ; Ahcy : adénosylhomocystéinase; Pccb : propionyl carboxylase ; Pah : phénylalanine hydoxylase ; Tat : tyrosine aminotransférase; Hgd: homogentisate dioxygénase; Hpd: hydroxylphénylpyruvate dioxygénase. G6P: glucose 6-phosphate; 3-PG: 3-phosphoglycérate ; 2-PG : 2-phosphoglycérate ; PEP : phosphoénolpyruvate ; Pyr : pyruvate ; OAA : oxaloacétate.

associé au fait que la concentration de la plupart des acides aminés étaient fortement diminuée dans le sérum et le muscle des animaux mutants, indiquait que chez les animaux déficients en LKBI dans le foie, l'extraction hépatique des acides aminés d'origine musculaire était accrue, conduisant à terme à la sarcopénie observée.
Nous avons confirmé que les aminotransférases, enzymes fréquemment utilisées dans les premières étapes de la synthèse de glucose à partir des acides aminés (Figure 1), relayaient en partie l'effet de la perte de LKBI dans le processus de néoglucogenèse dépendant des acides aminés, puisque leur inactivation génétique ou leur inhibition pharmacologique a permis un sauvetage partiel du phénotype des animaux mutants. En particulier, nous avons identifié le rôle de l'alanine-glyoxylate et sérine-pyruvate aminotransférase ( $A G X T$ ), une enzyme qui, outre son rôle dans la détoxification du glyoxylate, est impliquée dans la synthèse de glucose à partir de la sérine [8]. L'inactivation conjointe de $L K B I$ et 


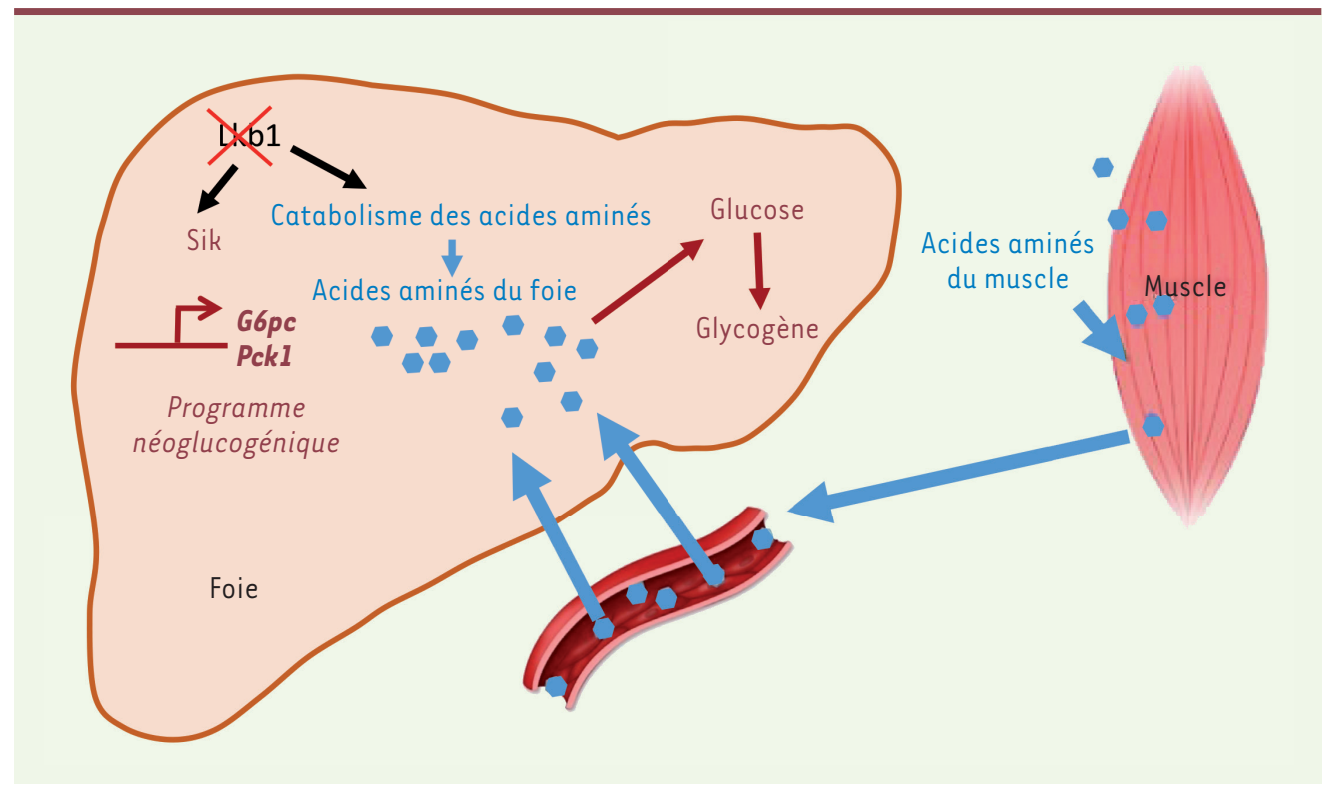

Figure 2. Rôle de LkbI comme suppresseur de la néoglucogenèse dépendante des acides aminés. Dans le foie de souris mutantes n'exprimant plus LKB 1 , le programme génique néoglucogénique ( $G 6 p c$ et $P c k 1$ ) via les kinases Sik est augmenté, ainsi que le catabolisme des acides aminés qui constituent des précurseurs de la synthèse de glucose. Les acides aminés provenant du muscle sont aussi utilisés comme précurseurs pour la synthèse de glucose dans le foie.

d'AGXt dans le foie a conduit à un retard dans le développement de la sarcopénie et de la cachexie des animaux mutants. Nous avons aussi identifié la protéine LKBI comme un acteur du contrôle de la néoglucogenèse post-prandiale. Ce processus, relativement peu étudié, a lieu au cours de la réalimentation, et permet de prendre en charge l'excès d'acides aminés présents dans le bol alimentaire, qui serait toxique s'ils n'étaient pas captés par le foie. Or contrairement au glucose, qui peut être stocké sous forme de glycogène, et aux acides gras, qui peuvent être stockés sous formes de triglycérides, la seule forme de stockage pour les acides aminés est le glucose. Ainsi la néoglucogenèse post-prandiale est essentielle pour stocker les acides aminés en excès provenant de l'alimentation. Nos résultats montrent que LKBI est un acteur majeur du contrôle de la néoglucogenèse non seulement au cours du jeûne, mais aussi en période post-prandiale. II est vraisemblable qu'une dérégulation de ce processus contribue à l'hyperglycémie post-prandiale des patients diabétiques de type 2.

En conclusion, nous avons identifié un nouveau rôle métabolique de la kinase LKBI hépatique dans le contrôle du métabolisme glucidique [7]. À jeun, LKBl permet de limiter l'extraction hépatique des acides aminés sériques et d'origine musculaire, et leur participation à la néoglucogenèse. À l'état nourri, LKBI limite également l'hyperglycémie qui serait provoquée par une activation trop forte de la néoglucogenèse post-prandiale, mécanisme physiologique de clairance des acides aminés alimentaires. Ainsi, LKBl apparaît comme un puissant gardien de la néoglucogenèse hépatique à partir des acides aminés (Figure 2). Une activation hépatique de LKBI constitue donc une piste thérapeutique séduisante pour contrôler l'hyperglycémie des patients diabétiques de type 2. $\diamond$

LKB1, a gatekeeper of amino-acid driven gluconeogenesis

\section{LIENS D’INTÉRÊT}

Les auteurs déclarent n'avoir aucun lien d'intérêt concernant les données publiées dans cet article.

\section{RÉFÉRENCES}

1. Sharabi K, Tavares CD, Rines AK, Puigserver P. Molecular pathophysiology of hepatic glucose production. Mol Asp Med 2015 ; 46 : 21-33.

2. Shulman GI, Landau BR. Pathways of glycogen repletion. Physiol Rev $1992 ; 72$ : 1019-35.

3. Alessi DR, Sakamoto K, Bayascas JR. LKB1-dependent signaling pathways. Annu Rev Biochem 2006 ; 75 : 137-63.

4. Ruderman NB, Carling D, Prentki M, Cacicedo JM. AMPK, insulin resistance, and the metabolic syndrome. J Clin Invest $2013 ; 123: 2764-72$.

5. Shaw RJ, Lamia KA, Vasquez D, et al. The kinase LKBl mediates glucose homeostasis in liver and therapeutic effects of metformin. Science $2005 ; 310$ : 1642-6.

6. Patel K, Foretz M, Marion A, et al. The LKBI-saltinducible kinase pathway functions as a key gluconeogenic suppressor in the liver. Nat Commun $2014 ; 5: 4535$.

7. Just PA, Charawi S, Denis RGP, et al. Lkbl suppresses amino acid-driven gluconeogenesis in the liver. Nat Commun $2020 ; 11: 6127$.

8. Rowsell EV, Snell K, Carnie JA, Al-Tai AH. Liver$\mathrm{L}$-alanine-glyoxylate and L-serine-pyruvate aminotransferase activities: an apparent association with gluconeogenesis. Biochem J 1969 ; 115 : 1071-3.

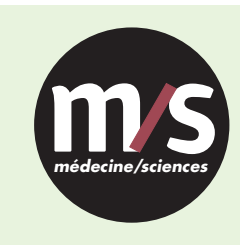

Tarifs d'abonnement $\mathrm{m} / \mathrm{s}-2021$

Abonnez-vous

à médecine/sciences
$>$ Grâce à $m / s$, vivez en direct les progrès des sciences biologiques et médicales

\section{Bulletin d'abonnement} page 938 dans ce numéro de $\mathrm{m} / \mathrm{s}$

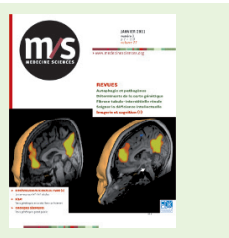

